\title{
Nosemosis en abejas melíferas y su relación con factores ambientales en Jalisco, México
}

\author{
Nosemosis in worker bees and their relationship with \\ environmental factors in Jalisco, Mexico
}

\author{
José María Tapia-Gonzáleza, Gustavo Alcazar-Ocegueraa , José Octavio Macías-Macías ${ }^{a *}$, Francisca Contreras- \\ Escareño ${ }^{b}$, José Carlos Tapia-Rivera ${ }^{a}$, Francisco Javier Chavoya-Moreno ${ }^{a}$, Juan Carlos Martínez-González ${ }^{c}$
}

\begin{abstract}
RESUMEN
La nosemosis es causada por el microsporidio Nosema spp que parasita el intestino medio de abejas melíferas adultas, es mortal en su forma aguda y ello ocasiona pérdidas económicas muy importantes al productor apícola. El conocimiento de la infección por $\boldsymbol{N}$. spp en abejas melíferas puede ayudar a prevenir la propagación o controlar la infección entre colonias. El objetivo del presente trabajo fue determinar la presencia e intensidad de infección de $\boldsymbol{N}$. spp en colonias de abejas melíferas y su relación con la temperatura, precipitación pluvial y altitud de esta región. Se obtuvieron 352 muestras de abejas Apis mellifera de colonias de ocho municipios del sur sureste de Jalisco. Para el diagnóstico se utilizó el método de macerado de abdómenes (Cantwell) y método de Jaycox para obtener el número de esporas de $\boldsymbol{N}$. spp por abeja. El $100 \%$ de las muestras fueron positivas; en $83.75 \%$ de ellas se presentó entre 1 a 5 millones de esporas por abeja. Se obtuvieron correlaciones débiles entre la intensidad de infección con la altitud $(r=-0.35)$, la temperatura $(r=0.44)$ y la precipitación pluvial $(r=-0.12)$, por lo que se sugiere que estos parámetros no influyen mayormente en la presencia de la enfermedad. Con los resultados obtenidos de nosemosis se puede sugerir la utilización de antibióticos o hacer control biológico, retirando cada año los panales más viejos en las colonias y el cambio anual de reinas.
\end{abstract}

PALABRAS CLAVE: Nosema spp, Apis mellifera, Prevalencia, Correlación ambiental, México.

\begin{abstract}
Nosemosis is caused by Nosema spp microsporidium that parasites the midgut of adult worker bees, it is mortal in its acute form and causes very important economic losses for the beekeeper. The knowledge of the infection caused by $\boldsymbol{N}$. spp in worker bees can help to prevent the spreading or control infection between colonies. The objective of the present work was to determine the presence and intensity of infection of $\boldsymbol{N}$. spp in worker bees' colonies and its relation with temperature, pluvial precipitation and altitude in this region. Samples of Apis mellifera workers $(n=352)$ were obtained from eight municipalities of south southwest of Jalisco. For the diagnosis, the abdomen tenderize method (Cantwell) was used and the Jaycox method to obtain the number of spores by bee. One hundred percent of the samples resulted positive, and $83.75 \%$ of them between 1 to 5 million spores of by bee was presented. Weak correlations were obtained between the intensity of infection with the altitude $(r=-0.35)$, the temperature $(r=0.44)$ and the pluvial precipitation $(r=-\mathbf{0 . 1 2})$, for what can be suggested that these parameters do not majorly influence the presence of the disease. The obtained results suggest that all workers bees' colonies of the sampled municipalities should be treated in a periodic way with antibiotics, or biologic control removing each year older honeycombs in the colonies and the annual change of queens.
\end{abstract}

KEY WORDS: Nosema spp, Apis mellifera, Prevalence, Environmental correlation, México.

Recibido el 8 de marzo de 2016. Aceptado el 1 de agosto de 2016.

a Departamento de Desarrollo Regional. Centro Universitario del Sur. Universidad de Guadalajara. Av. Enrique Arreola Silva no. 883. Cd. Guzmán, Jalisco. México.

${ }^{\mathrm{b}}$ Departamento de Producción Agrícola. Centro Universitario de la Costa Sur. Autlán, Jalisco, México.

${ }^{\text {c } F a c u l t a d ~ d e ~ I n g e n i e r i ́ a ~ y ~ C i e n c i a s ~ U A T . ~ C e n t r o ~ U n i v e r s i t a r i o ~ C i u d a d ~ V i c t o r i a ~ T a m a u l i p a s, ~ M e ́ x i c o . ~}$

*Autor de correspondencia: joseoc@cusur.udg.mx. 
La nosemosis que se presenta en abejas adultas, es ocasionada por dos especies de microsporidios llamados Nosema apis Zander y Nosema ceranae que afectan el intestino y ventrículo de las abejas, debilitan el organismo y acortan su periodo de vida ${ }^{(1-7)}$. Esta patología está ampliamente distribuida en todo el mundo; ocasionando debilitamiento del sistema inmune de las abejas, que predispone el ingreso de enfermedades virales que se relacionan con la actual desaparición de abejas a nivel mundial, denominado desorden del colapso de las colonias (CCD por sus siglas en inglés) ${ }^{(8)}$. Investigadores de algunos países culpan a $N$. apis en unión con $N$. ceranae, de la mortandad y despoblamiento de las colonias de abejas, y también indican que nosemosis puede ser un problema que restrinja el crecimiento de las poblaciones de abejas $^{(9,10)}$. En esta misma relación de presencia combinada de ambas especies de Nosema, se demostró en laboratorio que la presencia de ambas especies aumentaba el porcentaje de mortalidad, en relación a la infección por una sola especie ${ }^{(11)}$. En la actualidad existe un resurgimiento de la investigación sobre este parásito, sobre todo cuando se le asocia a la muerte masiva de colonias de abejas en Estados Unidos, Canadá y Europa(12,13).

Esta pérdida de colonias causada por la nosemosis provoca afectaciones económicas negativas al reducir la cantidad de colonias disponibles para la producción de los diversos productos apícolas, y para la polinización de cultivos $^{(14)}$. En México, la primera evidencia de la presencia de nosemosis fue en el año 1965 en el

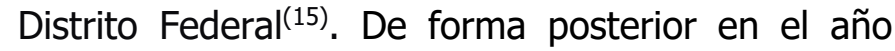
1982, se encontró 80 \% de infección, en 10 municipios de la zona centro de Jalisco(16). Para el año 1983 se determinó que $N$. apis estaba en $3.2 \%$ de las muestras de apiarios comerciales de Yucatán; en ese tiempo no se consideró un problema para la apicultura en México(17).

La nosemosis en México ha sido poco estudiada y no se le ha dado la importancia que requiere, sólo se han realizado algunos muestreos en los años 60s, estudios en tesis de licenciatura en los años 80s y algunos estudios en Yucatán, pero todos ellos indican la presencia de $N$. apis en diferente intensidad de infección en apiarios ${ }^{(13)}$. En la actualidad no se conoce cuál es la situación de la nosemosis y las relaciones que puede tener con las variables geográficas ambientales, en los apiarios del sur y sureste de Jalisco, México, por lo que el objetivo del presente trabajo, fue determinar la presencia, e intensidad de infección de $N$. spp en colonias de abejas melíferas, y determinar su relación con las variables geográficas ambientales en los municipios de la región sur-sureste del estado de Jalisco, México. Al obtener estos datos se podrán hacer recomendaciones para el control de dicha enfermedad.

El trabajo se realizó en ocho municipios que se seleccionaron de acuerdo a su clima (templado subhúmedo y cálido subhúmedo) con el fin de encontrar una relación biológica entre clima y presencia e intensidad de infección de Nosema. Los municipios se ubican en el occidente de México, dentro de las coordenadas geográficas; $19^{\circ} 24^{\prime}, 21^{\circ}$ $14^{\prime} \mathrm{N} ; 101^{\circ} 59^{\prime}, 1040$ 5' O. Los municipios de Tapalpa y Unión de Guadalupe tienen clima templado sub-húmedo y los municipios de Tecalitlán, Tamazula, Zacoalco de Torres, Sayula, Gómez Farías y Cocula, presentan clima cálido subhúmedo ${ }^{(18,19)}$.

Los municipios con clima cálido subhúmedo tienen una temperatura media anual mayor a $24^{\circ} \mathrm{C}$ y una temperatura media del mes más frío mayor a los $18^{\circ} \mathrm{C}$. El clima es semiseco, con otoño, invierno y primavera secos, y semicálidos, sin cambio térmico invernal bien definido y temperatura promedio de 24 ${ }^{\circ} \mathrm{C}^{(20)}$. El verano llega a ser algo caluroso con temperaturas de entre 38 y $39^{\circ} \mathrm{C}$, y en invierno llega a descender hasta $-7{ }^{\circ} \mathrm{C}$ en el valle. El régimen de lluvias se registra de junio a septiembre, con una precipitación de 578.7 a 1,768.4 mm, dependiendo el municipio. El promedio anual de días con heladas es de 120. Los vientos dominantes son en dirección del suroeste(20).

Los municipios con clima templado subhúmedo son semisecos, con otoño invierno y primavera húmedos, y semicálidos con un cambio térmico invernal bien definido; la temperatura media anual es de $18^{\circ} \mathrm{C}$, con máxima de $27.2^{\circ} \mathrm{C}$ y mínima $-5^{\circ} \mathrm{C}$ y altitud de 2,060 msnm en promedio, y con una precipitación media de 780 a $883.1 \mathrm{~mm}$ dependiendo del municipio. De acuerdo a esta 
distribución de climas, las temperaturas medias varían de manera importante según la altitud de las zonas, la humedad de la región y la evaporación. La época caliente del año corresponde a los meses de abril a septiembre. La temporada fría abarca los meses de octubre a marzo ${ }^{(19,20)}$. En estas regiones se encuentran matorrales, bosque, selva y suelo para agricultura. La vegetación se compone de especies de nogal, roble, encino, pino, cedro, mezquite, guamúchil, fresno, oyamel, sauce, madroño, huizache, palo dulce, granjeno, espino, jarrilla, ozote, tabachín, guaje, tasiste, amole, rosa morada, camichín, higuera, pitayo, fresno, madroño, maguey, frutales, guayabos, chirimoyos y tila; entre una gran variedad existente ${ }^{(20)}$.

En estas dos zonas geográficas se tomaron al azar muestras de 352 colonias de 135 apiarios sin importar su estado de fortaleza de población, durante la época de estiaje en los meses de marzo a mayo. El tamaño de la muestra se calculó para una población aproximada de 10,000 colmenas en los municipios evaluados que fueron considerados como estratos (cada municipio un estrato) para la comparación de medias, a un nivel de confianza del $95 \%$ y un poder estadístico del $80 \%$; como resultado se obtuvo un tamaño de 21 muestras por municipio, incluyendo $15 \%$ por pérdidas, mostrando una $\mathrm{n}=160$ colmenas; de ellas se realizó un muestreo aleatorio estratificado desproporcionado, sin embargo se obtuvieron y analizaron un total de 352 colmenas. Para la toma de las muestras se utilizaron frascos con $70 \mathrm{ml}$ de formol al $4 \%$ y para capturar las abejas, se tapó la piquera con una esponja. De esta manera se capturaron 25 abejas pecoreadoras que regresaban a la colmena.

Las muestras de abejas se procesaron en el laboratorio de Microbiología del Centro Universitario del Sur (CUSur de la Universidad de Guadalajara) en donde se utilizó la metodología de Cantwell y la técnica de Jaycox ${ }^{(21,22)}$ para la identificación y cuantificación del grado de infección de $N$. spp. Las esporas se observaron a 400 aumentos (seco fuerte) distinguiendo las esporas por ser corpúsculos brillantes y refringentes. Se utilizó la siguiente fórmula para determinar el número de esporas por abeja: ( $\mathrm{N}^{0}$ total de esporas contadas $/ 80$ ) x 4'000,000 $=\mathrm{N}^{\circ}$ de esporas por abeja. En los ocho municipios evaluados, la presencia de neosporosis se reportó en porcentaje de abejas con la enfermedad para cada muestra que fue positiva a las esporas, y la intensidad se clasificó de acuerdo a los resultados obtenidos en el conteo de esporas, tomando como base la clasificación de Jaycox ${ }^{(22)}$ (Cuadro 1). De cada apiario se registró la siguiente información: altitud (msnm), con un GPS (Sportrack-color Magellan), temperatura $\left({ }^{\circ} \mathrm{C}\right)$ y precipitación pluvial $(\mathrm{mm})$, tomadas de los datos registrados por CONAGUA ${ }^{(23)}$. Para determinar en los municipios la relación entre la intensidad (severidad) de la infección con las variables, altitud, temperatura ambiental y precipitación pluvial, se utilizó el coeficiente de correlación Pearson a través del programa estadístico SPSS(24).

En esta investigación, la presencia de $N$. spp fue positiva en el $100 \%$ de las muestras; $83.7 \%$ de las mismas presentaron una intensidad de infección ligera (de uno a cinco millones de esporas por abeja), muy ligera en el $13.3 \%$ (de cien mil a un millón de esporas por abeja), regular en el $2.6 \%$ (de cinco a diez millones) y semisevera $0.25 \%$ (de diez a veinte millones). En un trabajo similar ${ }^{(25)}$ en la zona sur de Jalisco, en el año 1996 se encontró infección muy ligera en $90 \%$ de las colonias evaluadas (cien mil a un millón de esporas por abeja). Es notable al comparar los dos trabajos, que $N$. spp ha aumentado la presencia e intensidad (severidad) de infección en las colonias de abejas en esta región conforme transcurren los años. Así lo demuestran más estudios, donde se observa el aumento de la presencia de Nosema y la severidad o intensidad de la infección; en el año 1989 se reportó $32.14 \%$ de presencia de $N$. apis en abejas de cinco estados de México(26). En 1991 se encontró $42.85 \%$ del mismo parásito en criaderos de abejas reinas de siete estados mexicanos ${ }^{(20)}$.

Cuadro 1. Intensidad de infección representada por el número de esporas (millones) por abeja

\begin{tabular}{cc}
\hline Intensidad de infección & Millones de esporas por abeja \\
\hline Nula & Menos de 0.01 \\
Muy ligera & $0.01-1.00$ \\
Ligera & $1.00-5.00$ \\
Regular & $5.00-10.00$ \\
Semisevera & $10.00-20.00$ \\
Severa & Más de 20.00 \\
\hline
\end{tabular}


Cuadro 2. Porcentaje de infección de N. spp por municipio de la zona sur sureste de Jalisco

\begin{tabular}{|c|c|c|c|c|c|c|c|}
\hline \multirow[b]{2}{*}{ Municipios } & \multirow[b]{2}{*}{ Zona climática } & \multirow[b]{2}{*}{$\begin{array}{l}\text { Colmenas } \\
\text { muestreadas }\end{array}$} & \multicolumn{5}{|c|}{ Intensidad } \\
\hline & & & Muy Ligera & Ligera & Regular & Semisevera & Severa \\
\hline Cocula & Cálido subhúmedo & 42 & 33 & 67 & & & \\
\hline Gómez Farías & Cálido subhúmedo & 15 & 20 & 80 & & & \\
\hline Sayula & Cálido subhúmedo & 52 & 12 & 79 & 9 & & \\
\hline Zacoalco & Cálido subhúmedo & 39 & 13 & 87 & & & \\
\hline Tamazula & Cálido subhúmedo & 93 & 1 & 87 & 10 & 2 & \\
\hline Tecalitlán & Cálido subhúmedo & 54 & 7 & 91 & 2 & & \\
\hline Tapalpa & Templado subhúmedo & 37 & 11 & 89 & & & \\
\hline Unión de Guadalupe & Templado subhúmedo & 20 & 10 & 90 & & & \\
\hline
\end{tabular}

En México y en otros países de América se ha encontrado que en abejas melíferas es más frecuente $N$. ceranae que $N$. apis. En 2011 se evaluaron 34 muestras de abejas en los estados de México, Distrito Federal, Morelos e Hidalgo, y encontraron $100 \%$ de presencia de ellas, $94.1 \%$ fue de $N$. ceranae y $5.9 \%$ de $N$. apisis); en Zacatecas México ${ }^{(28,29)}$ se evaluaron 299 colonias de abejas Apis mellifera, y a diferencia del anterior trabajo, encontraron una baja incidencia de $N$. spp, de $2.3 \%$. De forma similar al aumento a la presencia de Nosema en Quebec, Canadá(30) se encontró que en abejas melíferas, $75 \%$ de las muestras fueron positivas a $N$. ceranae sola o combinada con $N$. apis, y en $29.7 \%$ de muestras la presencia de $N$. apis sola; en el mismo país, en Alberta, en el año $2015^{(31)}$, encontraron a $N$. ceranae, en un rango de prevalencia del 41 al $91 \%$ y a $N$. apis entre 4 y $34 \%$, la infección mixta entre ambas fue de 5 a $25 \%$.

García(32) afirma que las colonias que poseen ambos parásitos tienen un riesgo de despoblación seis veces mayor que las demás colmenas, por lo que sería interesante hacer un muestreo en la zona sur sureste de Jalisco, para detectar la presencia de $N$. ceranae y así tener un diagnóstico más completo y sugerir acciones para su control. El problema de nosemosis puede ser grave en las zonas de estudio del presente trabajo, donde se encontró $87.75 \%$ de muestras positivas con infección (ligera) de $N$. spp. Al respecto se hace mención que las colmenas que rebasen el millón de esporas de $N$. apis deberán tratarse con el antibiótico fumagilina, ya que este parásito está afectando el adecuado desarrollo y la productividad de las mismas ${ }^{(15)}$. De forma similar en
Costa Rica(9), se reporta que los apiarios de San José, Alajuela y Guanacaste, estaban infectados en $67.5 \%$ por nosemosis, considerando que este grado de infección es alto, sugieren los autores el control con fumagilina para evitar el debilitamiento y muerte de la colonia(33). Sin embargo, otra opción para el control de la enfermedad puede ser el cambio anual de los tres bastidores más viejos, junto con el recambio anual o bianual de reinas (comunicación personal, Ernesto Guzmán Novoa).

En esta investigación, el municipio de Tamazula Jalisco, México, fue el único de ocho municipios evaluados donde se presentó intensidad de infección semisevera (de diez a veinte millones de esporas por abeja (Cuadro 2), 4 de los 8 apiarios de los ocho municipios evaluados rebasaron dos millones de esporas por abeja $(2 ' 285,562 \pm 702,575)$ (Cuadro 3 ). En relación a la intensidad o severidad de

Cuadro 3. Promedio (millones) de esporas de Nosemosis spp en abejas (muestra) evaluadas por municipio

\begin{tabular}{|l|c|c|}
\hline Municipio & $\begin{array}{c}\text { Total de } \\
\text { muestras }\end{array}$ & Promedio de esporas \\
\hline Tecalitlán & 54 & $2^{\prime} 504,630$ \\
\hline Tamazula & 93 & $3^{\prime} 297,590$ \\
\hline Sayula & 52 & $3^{\prime} 048,077$ \\
\hline Zacoalco & 39 & $2^{\prime} 947,436$ \\
\hline Cocula & 41 & $1^{\prime} 393,902$ \\
\hline Gómez Farías & 15 & $1^{\prime} 633,333$ \\
\hline Unión de Guadalupe & 20 & $1^{\prime} 632,500$ \\
\hline Tapalpa & 37 & $1^{\prime} 827,027$ \\
\hline Totales & 352 & $2^{\prime} 285,562 \pm 702,575$ \\
\hline
\end{tabular}


infección ligera que se encontró en todos los municipios evaluados en este trabajo, se hace notar que los municipios de Tapalpa y Unión de Guadalupe ubicados en zona de montaña (clima templado subhúmedo) presentaron diferencias de alrededor de 700 mil esporas menos en promedio por abeja, en comparación con las del valle con clima cálido subhúmedo (Gómez Farías, Tamazula, Tecalitlán, Zacoalco Cocula y Sayula) (Cuadro 3). Aun cuando la presente investigación se realizó en época de estiaje en los meses marzo-mayo, sin lluvias, en la estación climática de primavera, se esperaba que la intensidad de infección de $N$. spp en las colonias fuera más baja que en tiempo de lluvias o invierno. En relación a lo anterior, García ${ }^{(32)}$ menciona que el clima, la altitud y la humedad, entre otros factores, afectan la presencia de $N$. apis en las colonias de abejas; en Brasil ${ }^{(34)}$ reportaron la presencia de $N$. spp, en abejas africanizadas, y encontraron prevalencia más baja de nosemosis durante el invierno y primavera comparado con verano y otoño.

En el presente trabajo se encontraron correlaciones negativas débiles $(r=-0.35)(P=0.01)$, entre la altitud, con la intensidad de infección de $N$. spp; lo anterior sugiere poco efecto de altitud sobre el promedio de $1,000 \mathrm{~m}$, con menor infección con $N$ apis. En este mismo trabajo se encontró débil correlación negativa entre la intensidad de infección de $N$. apis $(r=-0.12)$ con los municipios que tienen más precipitación pluvial anual sobre el promedio (953 mm); esta relación no fue significativa $(P=0.16)$, pero la temperatura ambiental presentó una correlación media con la intensidad de infección de Nosema $(r=0.44)(P=0.01)$. Lo anterior sugiere que a medida que aumente la temperatura sobre el promedio de los $21^{\circ} \mathrm{C}$ en los municipios evaluados, aumentará la intensidad o severidad de la infección. Al respecto se conoce que $N$. ceranae es más patógena que $N$. apis y la proporción de $N$. ceranae es más alta en climas cálidos que en climas templados, mientras que $N$. apis prevalece más en climas fríos ${ }^{(35,36)}$. En este sentido se afirma que a $N$. ceranae, no le afecta el que llueva o no, que haga calor o frio y no es estacional, pero estas variables sí afectan a $N$. apis, que desaparece la mayoría del

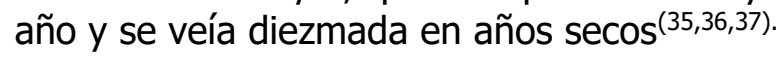

En conclusión, en el $100 \%$ de colonias de abejas melíferas en los municipios muestreados en el sur-sureste de Jalisco, se encontró la presencia del parásito $N$. spp. Se observa que aumentó la intensidad (severidad) de infección de muy ligera $(100,000$ a 1000,000$)$ en el año 1996 a ligera $(1000,000$ a 5000,000) en el año 2013. Las variables altitud, temperatura ambiental y precipitación de las dos zonas climáticas están correlacionadas de forma mediana o débil con la intensidad de infección de $N$. apis. Debido a la severidad de infección encontrada en los apiarios, sería interesante hacer un muestreo en esta zona para detectar la presencia y grado de infección de $N$. apis y $N$. ceranae y así tener un diagnóstico más completo y sugerir acciones para su control.

\section{LITERATURA CITADA}

1. Sepúlveda JMG. Apicultura. 2a ed. Barcelona, España: Aedos; 1980.

2. Fries I. Contribution to the study of Nosema disease (Nosema apis Z.) in honey bee (Apis mellifera L.) colonies. Sveriges Landbruksuniversitet, Institutionen för husdjurens utfodring och värd, Uppsala, Rapport 166, Sweaden. 1988.

3. Llorente MJ. Principales enfermedades de las abejas. 3a ed. Madrid, España. Servicio de extensión agrícola. 1990.

4. Polaino C. Manual práctico de apicultura. 3a ed. Madrid, España: Editora Cultural AC; 2006.

5. Bailey L. Patología de las abejas. 2a ed. España: Editorial Acribia; 1984.

6. Fritzcch, WB. Higiene y profilaxis en apicultura. 1a ed. España: Acribia; 1975.

7. Martínez LJF. Apicultura. 2a ed. Yucatán, México: Ediciones Martínez López. 1984.

8. Klee J, Besana AM, Genersch E, Gisder S, Nanetti A, Tam DQ. Widespread dispersal of the microsporidian Nosema ceranae, an emergent pathogen of the western honey bee, Apis mellifera. ] Invertebr Pathol 2007;96(1):1-10.

9. Calderón RA, Sanchez LA. Diagnóstico de enfermedades en colmenas africanizadas en Costa Rica: prevalencia y distribución de septiembre a noviembre del 2007. Agro Costa. 2011; 35(2):12-49. http: www.mag.go.cr/rev-agr/index.html. Consultado 18 Jun, 2015.

10. Forsgren E, Fries I. Comparative virulence of Nosema ceranae and Nosema apis in individual European honey bees. Vet Par 2010;70(3):212-217.

11. Geoffrey R, Shutler DWL, Burgher K, Rogers ELR. Infra-population and community dynamics of the parasites Nosema apis and Nosema ceranae, and consequences for honey bee (Apis mellifera), PLOS One 2014;7:14-19. 
12. Calderón RA, Sanchez LA, Yáñez O, Fallas N. Presence de Nosema ceranae in africanized honey bee colonies in Costa Rica. J Apic Res 2008; 47:328-329.

13. Guzmán-Novoa E. Que tan grave es la Nosemosis de abejas en México. Api 2009;(74):19-23.

14. Ritter WR. Enfermedades de las abejas. Zaragoza, España: Acribia; 2001.

15. Arriola GT, Fregoso SH. Enfermedades parasitarias diagnosticadas en abejas procedentes de criaderos de abejas reinas de México. VI Seminario Americano de Apicultura. Guadalajara, México. 1991:8182.

16. Chavira MBR. Identificación e incidencia de Nosema apis, Zander, en los apiarios de Jalisco [tesis licenciatura]. Guadalajara, Jalisco. Universidad de Guadalajara; 1982.

17. García MM, Quezada-Euan JJG. Distribución de Nosemiasis en apiarios comerciales de Yucatán, México. Api Mod 1993;2:17-23.

18. Plan Regional de Desarrollo. Región 05 Sureste de Jalisco. Comité Técnico de Planeación y Evaluación de Jalisco. Gobierno del Estado de Jalisco. 2012.

19. INEGI. Instituto Nacional de Estadística Geografía e Informática. Gobierno del estado de Jalisco, Sayula Jalisco. http://www. Jalisco. gob.mx/es/Jalisco/municipios. 2013. Consultado 19 Jun, 2015.

20. INEGI. Instituto Nacional de Estadística Geografía e Informática. Cuéntame de México. http://cuentame.inegi.org.mx/monografias/ informacion/jal/territorio/clima.aspx?tema=me. 2015. Consultado 19 Jun, 2015.

21. Molina PA. Patología apícola. 2a ed. El Salvador: OIRSA-SARH; 1990.

22. NOM-056-ZOO-1995. Especificaciones técnicas para las pruebas diagnósticas que realicen los laboratorios de pruebas aprobados en materia zoosanitaria. Diario oficial de la federación mexicana. 1999.

23. CONAGUA. Comisión Nacional del Agua. México. http://www. conagua.gob.mx/. 2015. Consultado 23 Jun, 2015.

24. SPSS. "Statistical Product and Service Solutions". Versión 20. IBM SPSS. http://www.softonic.com/s/spss-version-20. 2015. Consultado 27 Jul, 2015.

25. Nava BM. Nosemosis en abejas (Apis mellifera) en la región sur del estado de Jalisco, México [tesis licenciatura] Jalisco, México: Universidad de Guadalajara; 1996.
26. Martínez FJ, Medina ML, Catzín VA. Frecuencia de Varroa destructor, Nosema apis y Acaroptis woodi en colonias manejadas y enjambres de abejas (Apis mellifera) en Mérida, Yucatán, México. Rev Mex Cienc Pec 2011;2(1):25-38.

27. Guzmán-Novoa E, Hamiduzzaman MM, Arechavaleta VM, Pegah VE, Correa-Benítez A. Nosema ceranae has parasitized Africanized honey bees in Mexico since at least 2004. J Apic Res 2011;50(2):167-169.

28. Medina FCA, Guzmán-Novoa E, Espinosa MLG, Uribe RJL, Gutiérrez LR, Gutiérrez PFJ. Frecuencia de varroosis y nosemosis en colonias de abejas melíferas (Apis mellifera) en el estado de Zacatecas, México. Rev Chap 2014;(20)3:23-28.

29. Medina FCA. Principales enfermedades que afectan a las abejas melíferas (Apis mellifera) [tesis licenciatura]. Zacatecas, México: Universidad Autónoma de Zacatecas; 2014.

30. Copley TR, Chen H, Giovenazzo P, Houle E, Jabaji SH. Prevalence and seasonality of Nosema species in Québec honey bees. Can Entom 2012; 144(4):577-588.

31. Berna E, Guzmán-Novoa E, Hamiduzzaman MM, Eccles L, Lacey BA, Ruiz PR, Nasr M. Higher prevalence and levels of Nosema ceranae than Nosema apis infections in Canadian honey bee colonies. Par Res 2016; 115:175-181.

32. García SJ. Nosema el asesino de las abejas. Apicultura. http: miscelánea-noticias.blog.sport.mx/2007/09/n. 2007. Consultado 28 Jul, 2015.

33. Mendoza Y, Harriet J, Campa KJ, Ramallo H, Díaz G, Cetti S, Invernizzi C. Control de Nosema ceranae en colonias de abejas (Apis mellifera) en forestaciones de Eucalyptus grandis. Agroc 2013;(17):1-9.

34. Santos LG, Alves MLTMF, Message D, Pinto FA, Silva MVGB, Teixeira EW. Honey bee health in apiaries in the vale do Paraíba, São Paulo State, Southeastern. Brazil Soc 2014;61(3):307-312.

35. Fries I. Nosema ceranae in European honey bees (Apis mellifera). J Invert Pathol 2010;103:73-79.

36. Higes PM. Primera detección de Nosema ceranae, un nuevo microsporidio parásito, en el hospedador Apis mellifera, en España. http: www.agrodigital.com. 2005. Consultado 2 Jul, 2015.

37. Alatorre RR. Enfermedades de las abejas (Apis mellifera) L. Simposio Nacional de Apicultura. Oaxtepec, Morelos, México. 1989:107-122. 